\title{
Proper treatment of breast angiosarcoma-mastectomy or breast conserving surgery?
}

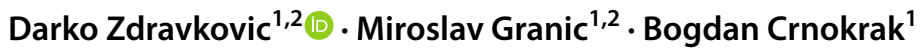

Received: 19 November 2019 / Accepted: 8 December 2019 / Published online: 17 December 2019

(c) Springer Science+Business Media, LLC, part of Springer Nature 2019

\section{Introduction}

We have read with great interest the article "Primary and secondary breast angiosarcoma: single center report and a meta-analysis" published by Abdou et al. [1]. Firstly, we would like to congratulate the authors on this comprehensive article and huge experience. However, we would like to comment some facts concerning the surgical treatment of breast angiosarcoma.

Complete surgical excision with optimal margins is a cornerstone of surgical treatment in breast angiosarcoma patients. There are two surgical options: mastectomy and breast conserving surgery (BCS). Authors concluded that there were no statistically significant outcomes concerning recurrence-free survival or overall survival comparing mastectomy to BCS. In our opinion, BCS should be considered only in patients with breast angiosarcoma with small size tumors [2]. Besides, BCS is a rather challenging approach in the combination of big tumors and small- or medium-sized breasts. The average tumor size in this paper was $6.9 \mathrm{~cm}$. Also, we would like to point out that the tumor grade is rather an important factor of an unfavorable prognosis. More than sixty percent of patients in this paper had a high tumor grade. Finally, unclear data regarding multifocality and a small number of patients should also be considered in making conclusions.

Further studies with a larger number of patients, using univariant and multivariant analysis, should be performed in order to achieve adequate conclusions [3].

Darko Zdravkovic

drdarkozdravkovic@gmail.com

1 University Medical Center „Bezanijska Kosa“, Belgrade, Serbia

2 Faculty of Medicine, University of Belgrade, Bezanijska kosa street bb, 11080 Belgrade, Serbia
Acknowledgements The authors have not received any grants.

\section{Compliance with ethical standards}

Conflict of interest The authors declare that they have no conflict of interest.

Ethical approval This article complies with the ethical rules applicable for this journal.

Human and animal rights This article does not contain any studies with human participants or animals performed by any of the authors.

\section{References}

1. Abdou Y, Elkhanany A, Attwood K, Ji W, Takabe K, Opyrchal M (2019) Primary and secondary breast angiosarcoma: single center report and a meta-analysis. Breast Cancer Res Treat 178(3):523-533

2. Taffurelli M, Pellegrini A, Meattini I et al (2019) Secondary breast angiosarcoma: a multicentre retrospective survey by the National Italian Association of Breast Surgeons (ANISC). Breast 45:56-60

3. McClelland S III, Hatfield J, Degnin C, Chen Y, Mitin T (2019) Extent of resection and role of adjuvant treatment in resected localized breast angiosarcoma. Breast Cancer Res Treat 175(2):409-418

Publisher's Note Springer Nature remains neutral with regard to jurisdictional claims in published maps and institutional affiliations. 